\title{
Multifunctional factor ENY2 is associated with the THO complex and promotes its recruitment onto nascent mRNA
}

\author{
Daria V. Kopytova, ${ }^{1}$ Anastasija V. Orlova, ${ }^{1}$ Alexey N. Krasnov ${ }^{1,2}$ Dmitriy Ya. Gurskiy, ${ }^{1}$ \\ Julia V. Nikolenko, ${ }^{1}$ Elena N. Nabirochkina, ${ }^{1}$ Yulii V. Shidlovskii, ${ }^{1,2,4}$ and Sofia G. Georgieva ${ }^{1,3,5}$ \\ ${ }^{1}$ Department of Regulation of Gene Expression, Institute of Gene Biology, Russian Academy of Sciences, Moscow 119334 Russia; \\ ${ }^{2}$ University of Oslo, Centre for Medical Studies in Russia, Moscow 119334, Russia; ${ }^{3}$ Department of Transcription Factors, \\ Institute of Molecular Biology, Russian Academy of Sciences, Moscow 119991, Russia
}

\begin{abstract}
Metazoan E/y)2/ENY2 is a multifunctional protein important for transcription activation and mRNA export, being a component of SAGA/TFTC and the mRNA export complex AMEX. Here, we show that ENY2 in Drosophila is also stably associated with THO, the complex involved in mRNP biogenesis. The ENY2-THO complex is required for normal Drosophila development, functioning independently on SAGA and AMEX. ENY2 and THO arrive on the transcribed region of the hsp70 gene after its activation, and ENY2 plays an important role in THO recruitment. ENY2 and THO show no direct association with elongating RNA polymerase II. Recruitment of ENY2 and THO occurs by their loading onto nascent mRNA, apparently immediately after its synthesis, while the AMEX component Xmas-2 is loaded onto mRNA at a later stage. Knockdown of either ENY2 or THO, but not SAGA or AMEX, affects the processing of the transcript's 3' end. Thus, ENY2, as a shared subunit of several protein complexes governing the sequential steps of gene expression, plays an important role in the coordination of these steps.
\end{abstract}

[Keywords: THO; mRNA export; mRNP formation; gene expression; protein complex; ENY2]

Supplemental material is available at http://www.genesdev.org.

Received July 22, 2009; revised version accepted November 6, 2009.

$\mathrm{E}(\mathrm{y}) 2$, the protein encoded by the $e(y) 2$ gene of Drosophila melanogaster (Georgiev and Gerasimova 1989), was first characterized as a transcription activator essential for fly development (Georgieva et al. 2001). The $\mathrm{E} \mid \mathrm{y} / 2$ protein is evolutionarily conserved from humans to yeast, where its homolog was designated Sus1 (Georgieva et al. 2001; Rodriguez-Navarro et al. 2004; Krasnov et al. 2005). The term ENY2, proposed for the human homolog (Zhao et al. 2008), will be used further in this study, instead of $\mathrm{E}(\mathrm{y}) 2$, to introduce a common term for metazoan proteins.

ENY2 is a small protein that plays an important role in transcription activation on a chromatin template, being a subunit of the SAGA/TFTC-type histone acetylation complex in Drosophila (Georgieva et al. 2001; MM Kurshakova et al. 2007) and yeast (Rodriguez-Navarro et al. 2004). In TFTC, ENY2 is a component of the subcomplex involved in deubiquitination of histone $\mathrm{H} 2 \mathrm{~B}$ (Kohler et al. 2006; Zhao et al. 2008).

Drosophila ENY2, together with Xmas-2, was also shown to be a component of AMEX, the complex essential for mRNP export from the nucleus and for anchoring

Corresponding authors.

${ }^{4}$ E-MAIL yul@genebiology.ru; FAX 007-499-135-4105.

${ }^{5}$ E-MAIL sonjag@molbiol.edu.ru; FAX 007-499-135-4105.

Article is online at http://www.genesdev.org/cgi/doi/10.1101/gad.550010. transcriptionally active genes to the nuclear pore complex (NPC) (MM Kurshakova et al. 2007). Yeast Sus1 interacts with an NPC-associated mRNA export complex termed TREX-2 (Fischer et al. 2002) that also contains Thp1, Cdc31, and Sac3, a homolog of Drosophila Xmas-2 (Rodriguez-Navarro et al. 2004; Jani et al. 2009), and is responsible for gene gating to the NPC (Cabal et al. 2006). These facts suggest that the function of ENY2/Sus1 is evolutionarily conservative.

Furthermore, ENY2 is involved in the functioning of the $\mathrm{Su}(\mathrm{Hw})$ insulator, binding to the $\mathrm{Su}(\mathrm{Hw})$ protein and accounting for the barrier function of this insulator $(M$ Kurshakova et al. 2007). Thus, ENY2 is a multifunctional protein connected with the control of gene expression, and plays an important role in development. It may well be that ENY2 also plays a certain role in integrating several steps of transcription elongation and mRNA export. To approach this problem, we continued our studies on ENY2 functional complexes and found that ENY2 also interacted with the THO complex.

The yeast THO complex was shown to be involved in transcription elongation, mRNA biogenesis, and mRNA export (Chavez et al. 2000; Jimeno et al. 2002; Strasser et al. 2002). THO components were biochemically copurified with the mRNA export factors Sub2p and Yralp within 
a bigger complex termed TREX (Strasser et al. 2002). THO is recruited cotranscriptionally to active chromatin through interaction with the transcription machinery and is thought to facilitate the subsequent recruitment of Sub2p and Yra1 to nascent mRNAs (Zenklusen et al. 2002; Abruzzi et al. 2004; Jimeno et al. 2006). The results of chromatin immunoprecipitation (ChIP) in combination with an RNase assay indicate that yeast THO interacts with DNA but not nascent RNA during transcription elongation (Abruzzi et al. 2004). Recent studies have shown that defective $\mathrm{THO} / \mathrm{Sub} 2 \mathrm{p}$ function affects mRNA 3 '-end processing (Saguez et al. 2008), and that THO and Sub2p participate in an mRNP remodeling event that follows mRNA 3'-end processing (Rougemaille et al. 2008).

Drosophila and human THO complexes have also been purified (Rehwinkel et al. 2004; Masuda et al. 2005). The Drosophila THO contains THO2, HPR1, and three proteins (THOC5, THOC6, and THOC7) that have orthologs in metazoans but not in budding yeast. UAP56 and REF1/ Aly, Drosophila homologs of Sub2p and Yralp TREX subunits, were not found in the purified complex, indicating their low affinity to THO. Unexpectedly, while the export of heat-shock mRNPs was shown to depend on THO function under stress, the vast majority of mRNAs proved to be transcribed and exported independently of THO in cell culture (Rehwinkel et al. 2004).

In contrast to the yeast complex, which is recruited cotranscriptionally, human TREX was found to be recruited to the transcribed gene via interaction with the mRNA processing machinery (Masuda et al. 2005). It was subsequently shown that TREX is recruited close to the 5 ' end of mRNA. Its recruitment requires the cap structure and the cap-binding protein CBP80 (Cheng et al. 2006). The THOC5 subunit of human THO binds hsp70 mRNA and functions in $h s p 70$ mRNP export via interaction with the export receptor Tap-p15 (Katahira et al. 2009).

Here, we show that Drosophila ENY2 copurifies with THO complex from the nuclear extract. ENY2 and THO colocalize on polytene chromosomes of Drosophila larvae, and the combination of weak mutations in the $e(y) 2$ gene and in one of the genes encoding THO components has a strong synergistic effect. ENY2 and THO are recruited to the transcribed region of the $h s p 70$ gene upon transcription activation and are cotranscriptionally loaded onto nascent mRNA. The presence of ENY2 is essential for THO recruitment to the transcribed region of the gene. The depletion of ENY2 and THO in Schneider 2 (S2) cells impairs the processing of the 3 ' end of nascent mRNA. The ENY2-THO complex functions independently on SAGA and AMEX. In summary, ENY2 participates in the basic events in the fate of nuclear mRNA: transcription activation, mRNP formation, and mRNA processing and export.

\section{Results}

ENY2 copurifies with the THO complex separated from SAGA and AMEX

As shown in our previous study (MM Kurshakova et al. 2007), ENY2 is a component of the SAGA/TFTC complex and the mRNA export complex AMEX. However, during size fractionation of Drosophila embryonic nuclear extract on a Superose 6 gel filtration column, ENY2 was eluted in different fractions, as at least three major peaks with apparent molecular weights of $\sim 2.0,1.5$, and 0.7 $\mathrm{MDa}$ (Fig. 1A). Treating the initial extract with DNase I or RNase did not change the ENY2 migration profile, which excluded the possibility of ENY2 association with other proteins through DNA or RNA fragments.

To determine the polypeptide composition of individual ENY2-containing complexes, we performed their stepwise purification from the embryonic nuclear extract (Fig. 1B). The 0.7-MDa complex was separated during fractionation on a Monos column, while two highmolecular-weight complexes were eluted close to each other at all fractionation steps. At the last step, the partially purified ENY2-containing material was loaded onto a Superose 6 column (Fig. 1A). It is noteworthy that the positions of ENY2 in it were practically the same as in the crude extract (Fig. 1A, two top panels), indicating the stability of the complexes during purification. Fractions corresponding to different ENY2 peaks were collected, and ENY2-associated proteins were immunoprecipitated with anti-ENY2 antibodies from each pool of fractions and identified with MALDI-TOF MS (Fig. 1C).

We found that the 1.5-MDa complex corresponds to SAGA (the molecular weight of this complex is in agreement with published data [Kusch et al. 2003]); the 0.7-MDa complex corresponds to AMEX (these interactions will be described elsewhere) (MM Kurshakova et al. 2007). Analysis of the 2.0-MDa ENY2-containing complex revealed a complete set of subunits of the THO complex-HPR1, THO2, THOC5, THOC6, and THOC7identical to that isolated previously (Rehwinkel et al. 2004). We did not detect TREX components Aly/Yra1 and UAP56/sub2, in agreement with the data that their association with the core THO complex is unstable (Rehwinkel et al. 2004). The presence of low-molecularweight ENY2 in the preparation was checked using Western blotting (Fig. 1C, bottom panel). Our preparation also contained several architectural proteins and subunits of the Brahma chromatin remodeling complex (Mohrmann and Verrijzer 2005). The reliability and functional importance of these interactions are now being analyzed.

To study the interaction of ENY2 with THO, polyclonal antibodies against the conservative THO component HPR1 and metazoan-specific THOC5 were raised in rabbits. Their specificity is shown in Figure 1D. These antibodies were used to analyze the Superose 6 fractions of crude nuclear extract for the distribution of THO (Fig. 1A). The migration profiles of HPR1 and THOC5 were identical, and the THO complex was eluted in fractions corresponding to proteins with molecular weights between $2 \mathrm{MDa}$ and $700 \mathrm{kDa}$; i.e., higher than expected from THO composition ( $400 \mathrm{kDa})$. This could be due to multimerization of THO complex subunits. We also observed that the THO complex is stable, since its gel filtration profile remained almost unchanged after several purification steps (Fig. 1A, bottom panels). 
A

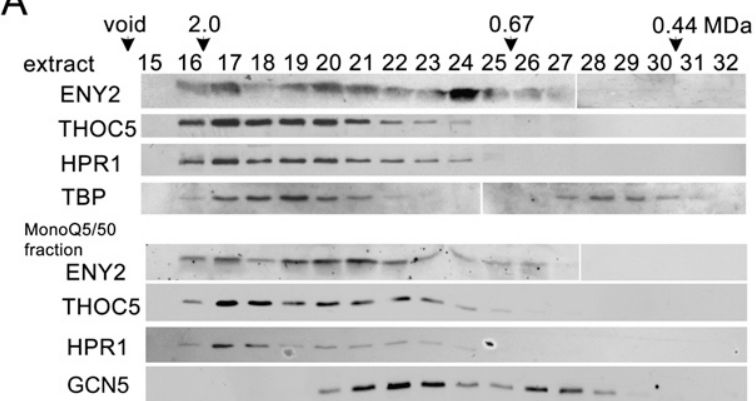

C

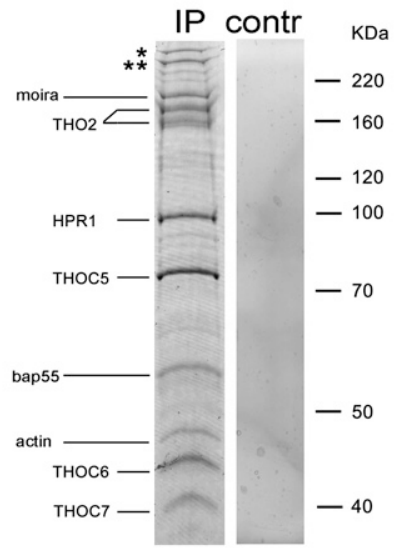

extract IP control

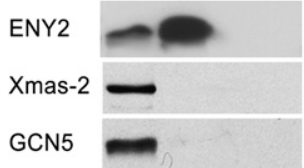

B

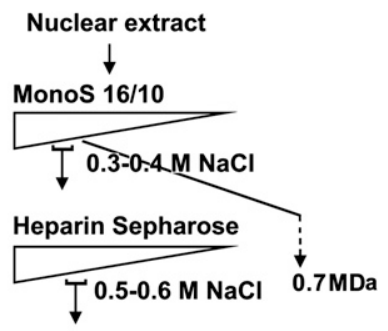

MonoQ 5/50

D

THOC5 HPR1

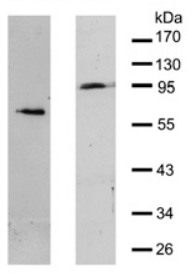

$E$

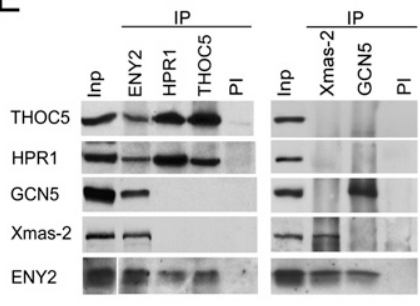

Figure 1. ENY2 is associated with THO complex. $(A)$ ENY2 is present in several stable complexes. A crude embryonic extract treated with DNase I and RNase was fractionated on a Superose 6 gel filtration column. The fractions were collected and analyzed for the presence of ENY2, THOC5, and HPR1 by Western blot analysis. Fraction numbers and the void volume are indicated. The distribution of TBP, which migrates with two characteristic peaks (Demeny et al. 2007), is shown to verify the quality of fractionation. The bottom panels represent Superose 6 fractionation of a partially purified preparation of high-molecular-weight ENY2-containing complexes. The profile of SAGA subunit GCN5 is shown for comparison with those of THO and ENY2. (B) Scheme of purification of the ENY2-THO complex. At each step, proteins were eluted with a $\mathrm{NaCl}$ gradient, and the fractions were analyzed by Western blotting for the presence of ENY2. The peak ENY2-containing fractions (corresponding $\mathrm{NaCl}$ concentrations are indicated) were collected and loaded onto the next column. Note that the 0.7-MDa complex was separated from high-molecular-weight complexes during fractionation on MonoS. After Superose 6 fractionation, the material was loaded onto an immunosorbent carrying antibodies against ENY2, washed with a buffer containing $1 \mathrm{M} \mathrm{NaCl}$, and eluted with acid glycine. $(C)$ The preparation of the 2.0-MDa ENY2-containing complex. Proteins eluted from the immunosorbent were resolved by $9 \%$ SDS-PAGE, stained with Coomassie, and analyzed by mass spectrometry. Bands indicated by a single asterisk $\left({ }^{\star}\right)$ and a double asterisk $\left({ }^{\star \star}\right)$ contained $\alpha$-spectrin and myosin, respectively. The control immunoprecipitation of the same material with preimmune IgG is shown on the right. The bottom panels show Western blot analysis of the nuclear extract, 2-MDa ENY2-containing preparation, and control immunoprecipitation for the presence of ENY2, Xmas-2 subunit of AMEX, and GCN5 subunit of SAGA. $(D)$ Specificity of antibodies against HPR1 and THOC5 as tested by Western blot analysis on the nuclear extract from Drosophila embryos. (E) Co-IP of ENY2 with THO components. Immunoprecipitation from the nuclear extract was performed with antibodies against ENY2, subunits of THO (THOC5 and HPR1), SAGA (GCN5), and AMEX (Xmas-2), or with a preimmune serum (PI). Although ENY2 is associated with all factors examined, no co-IP was detected for THO and SAGA or AMEX. Equal amounts of the extract (Inp) and precipitated proteins (IP) were analyzed.

Immunoprecipitation from the fractions showed that ENY2 was associated with only the high-molecularweight portion of THO in fractions 16 and 17 (Supplemental Fig. 1).

Stable association of ENY2 with THO was corroborated by the results of immunoprecipitation from the DNase- and RNase-treated embryonic nuclear extract with antibodies against ENY2, HPR1, or THOC5 (Fig. 1E). Antibodies against ENY2 did not completely deplete the extract of THO components, confirming the association of ENY2 with a portion of THO.

We analyzed the potential interaction of the ENY2THO complex with two other ENY2-containing complexes, SAGA and AMEX (subunits of the complexes 
considered in this study are listed in Supplemental Table 1). The AMEX complex was separated from THO at the first step of purification (Fig. 1B). Although ENY2-SAGA followed ENY2-THO through the first three steps of purification, they were clearly separated on the gel filtration column: GCN5 (a component of SAGA) was absent from high-molecular-weight fractions containing the ENY2THO complex (Fig. 1A, bottom panels). Western blot analysis of the ENY2-THO preparation did not reveal the presence of any residual amounts of SAGA and AMEX subunits (Fig. 1C, bottom panel). Likewise, no association of THO with SAGA or AMEX subunits was detected in immunoprecipitation experiments (Fig. 1E), with this result being independent of the stringency of washing (Supplemental Fig. 2).

Thus, biochemical experiments showed that the ENY2-THO complex exists as a stable entity distinct from SAGA and AMEX.

THO interacts with ENY2 in vivo and, compared with SAGA, has a different effect on the phenotypic traits of ENY2 mutants

To test whether ENY2 and THO are colocalized in vivo, we stained salivary gland polytene chromosomes with anti-THOC5 and anti-ENY2 antibodies (Fig. 2A). The

A

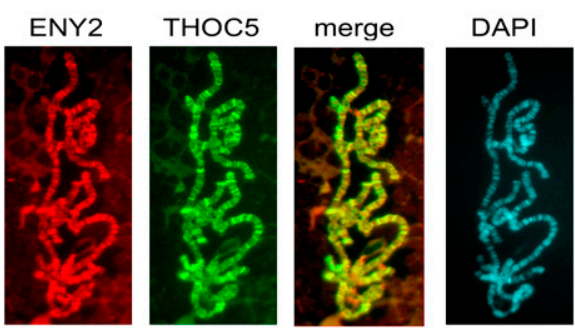

B

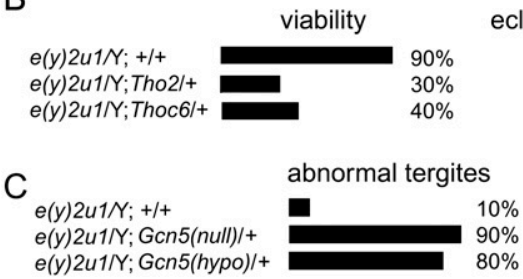

Figure 2. ENY2 interaction with THO complex in vivo. $(A)$ ENY2 colocalizes with THOC5 at many sites on polytene chromosomes from Drosophila salivary glands. Polytene chromosomes stained with antibodies against ENY2 and THOC5, their merged image, and DAPI staining are shown. (B) ENY2 interacts with THO during Drosophila development. Mutations tho2 and thoc6 in the heterozygous state result in decreased viability and eclosion delay in males hemizygous for the $e(y) 2^{u 1}$ mutation, but have no effect on their morphology. (C) ENY2 interacts with GCN5 during Drosophila development. Introduction of GCN5-null or hypomorphic allele in the heterozygous state in males hemizygous for the $e(y) 2^{u 1}$ mutation seriously aggravates abnormal development of tergites 9 and 10, with the mutant phenotype becoming more prominent (see Supplemental Fig. 4).
anti-THOC5 antibody stained a large number of actively transcribed loci (Fig. 2A, cf. THO and DAPI staining), and almost all of these loci were also stained with the antiENY2 antibody (see "merge" in Fig. 2A). This is evidence for in vivo THO and ENY2 colocalization on chromosomes. The presence of THOC5 and ENY2 at multiple chromosome sites indicates that they are essential for the expression of many Drosophila genes.

To estimate the relative abundance of ENY2, THO, and SAGA in different loci of the genome, we performed triple colocalization of these factors on polytene chromosomes (Supplemental Fig. 3). Although ENY2 and HPR1 are distributed genome-wide, some of the corresponding loci contain almost no SAGA component Ada2b (Kusch et al. 2003), which is evidence that THO and ENY2 may be abundant on SAGA-free genes.

We then examined whether ENY2 genetically interacts with THO components during Drosophila development. We previously described the $e(y) 2$ gene mutation, $e(y) 2^{u 1}$, that is caused by insertion of the Stalker mobile element into the noncoding region of the gene and reduces fourfold the level of its transcription (Georgieva et al. 2001). Hemizygous $e(y) 2^{u 1} / Y$ males have almost normal viability $(90 \%)$, show a slight delay of eclosion, and develop to the adult stage. About $10 \%$ of adult males are sterile and have abnormal morphology of tergites 9 and 10 (Supplemental Fig. 4).

The $e(y) 2^{u 1}$ flies were crossed with available strains of flies bearing tho mutations tho2 and thoc6, caused by $P$-element insertion into noncoding regions of the genes. Flies homozygous for these mutations did not display any mutant phenotype. However, males hemizygous for $e(y) 2^{u 1}$ and heterozygous for either the tho2 or thoc6 mutation (Fig. 2B) were characterized by low viability [two-thirds lower than that of $e(y) 2^{u 1} / Y$ males] and considerably delayed eclosion. Thus, even when heterozygous, the mutations reducing the level of THO components aggravated the weak mutation of the $e(y) 2$ gene. In the case of THO mutations, we observed no increase in the number of flies with abnormal tergites.

Next, we estimated if the interaction of the $e(y) 2^{u 1}$ mutation with mutations in the GCN5 component of SAGA would have the same effect on the phenotype of flies. The introduction of GCN5 mutations (null allele GCN5 or hypomorphic allele GCN5) (Carre et al. 2005) caused no change in the viability or eclosion time of males hemizygous for $e(y) 2^{u 11}$ and heterozygous for either of the GCN5 alleles. However, it dramatically enhanced the morphological manifestation of the $e(y) 2^{u 1}$ mutation: About $80 \%-90 \%$ of males hemizygous for $e(y) 2^{u 1}$ and heterozygous for either of the GCN5 mutated alleles had abnormal tergites, with the mutant phenotype being more prominent (Fig. 2C; Supplemental Fig. 4). Thus, mutations of either GCN5 or THO aggravated different phenotypic manifestations of the $e(y) 2^{u 1}$ mutation, suggesting that the ENY2-THO and ENY2-SAGA complexes have independent molecular functions.

In general, these experiments confirm that ENY2 and THO cooperate in fly development, with this cooperation being independent of SAGA. 


\section{Both ENY2 and THO are recruited onto the transcribed region of hsp70 following transcription activation}

Next, we studied the interaction of ENY2 with the THO complex in the control of individual gene expression using the example of the Drosophila gene hsp70. This gene was chosen because the Drosophila THO is required for the nuclear export of $h s p 70$ mRNA under heat stress (Rehwinkel et al. 2004). The SAGA complex controls hsp70 transcription under normal conditions and after heat shock (Lebedeva et al. 2005). Both the ENY2 and Xmas-2 subunits of AMEX were shown to be absolutely necessary for hsp70 mRNA export (MM Kurshakova et al. 2007).

The hsp70 gene cluster contains six almost identical copies of the intronless hsp 70 gene that are synchronously activated by heat shock. The distribution of ENY2, THO (HPR1 and THOC5), and RNA polymerase II (Pol II) along the hsp70 genes in S2 cells was analyzed under both nonheat-shock and heat-shock conditions by ChIP using 13 pairs of primers covering the whole $h s p 70$ region (Fig. 3A). Under non-heat-shock conditions, ENY2 was detected on the hsp70 promoter, similarly to the GCN5 and Ada2b components of SAGA (Lebedeva et al. 2005). Pol II was also present in the promoter region, as was observed previously (Rasmussen and Lis 1993). In contrast, HPR1 and THOC5 were not found on inactive hsp70. This result was confirmed by staining polytene chromosomes of Drosophila larvae with antibodies against THOC5 (Fig. 3B). Without heat shock, no immunostaining was observed in the region of hsp70 loci (87A-87B).

Under heat shock, in accordance with the high level of hsp70 transcription, the amount of Pol II strongly increased in the transcribed region. ENY2 was again detected on the hsp70 promoter, with its peak being shifted slightly to the transcribed region, similarly to the peak of Pol II. In addition, the second broad peak of ENY2 was observed in the transcribed region. Components of the THO complex were also detected on hsp70 in both ChIP and polytene chromosome staining experiments (Fig. 3A,B). Interestingly, THO subunits, similar to ENY2, displayed two peaks. The sharp peak was observed immediately downstream from the promoter, while the second broad peak covered the whole transcribed region of hsp 70. Thus, both ENY2 and THO were recruited onto the $h s p 70$ transcribed region upon transcription activation, and the distribution of HPR1 and THOC5 generally coincided with that of ENY2.

We also checked the presence of the Xmas-2 component of AMEX (MM Kurshakova et al. 2007) on the hsp70 gene. However, Xmas-2 was not detected using conventional ChIP (data not shown). This fact was apparently explained by low efficiency of Xmas-2 cross-linking with chromatin rather than by the failure of antibodies to react with this component, since they proved to be reactive in immunoprecipitation (MM Kurshakova et al. 2007) and RNA immunoprecipitation experiments (see below).

\section{ENY2 is crucial for THO recruitment to the hsp70 gene}

To find out if ENY2 participates in THO complex recruitment to the gene, we performed ENY2 knockdown
A
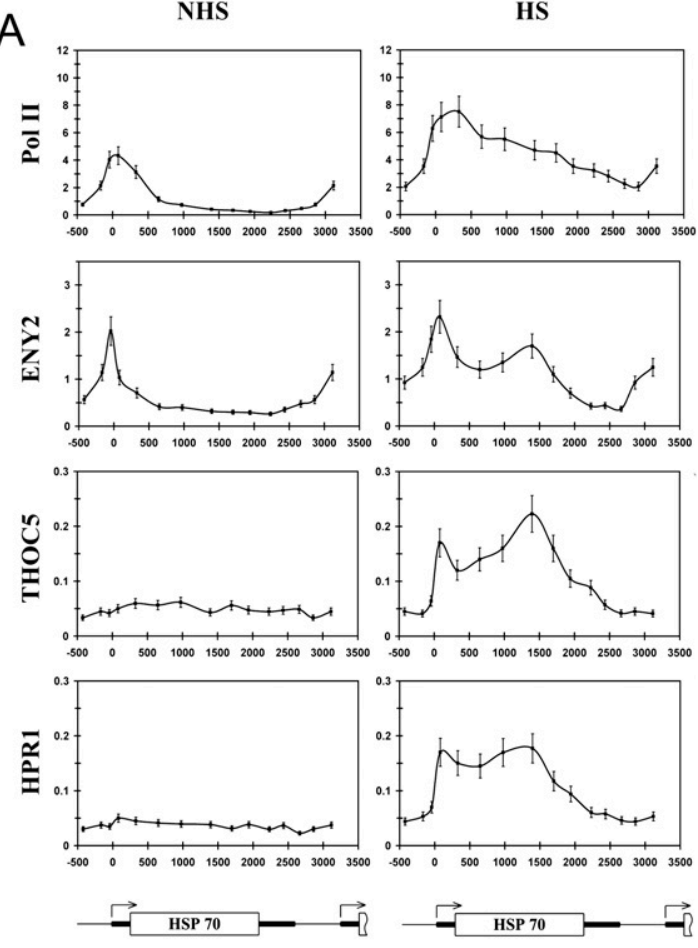

B

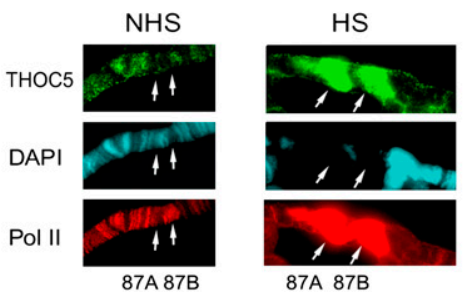

Figure 3. ENY2 and THO are recruited into the transcribed region of $h s p 70$ after transcription activation. $(A)$ The distribution of Pol II, ENY2, THOC5, and HPR1 on the hsp70 gene before (NHS [non-heat shock]) and after (HS [heat shock]) gene activation assayed by ChIP. The levels of these proteins were measured by ChIP at 13 points. The results of ChIP are shown as the percentage of input, with the baseline level varying from $0.02 \%$ to $0.05 \%$ at different points. The arrow indicates the transcription start site, the black bars are untranslated regions, and the white box is the ORF within the gene. $(B)$ THO is recruited to heat-shock-induced puffs (87A and 87B) on polytene chromosomes of Drosophila larvae. Polytene chromosomes were stained with antibodies against THOC5 and Pol II and with DAPI.

by RNAi in S2 cells and studied the presence of THO on hsp70 after heat shock (Fig. 4A). The level of ENY2 in cells was reduced $\sim 20$-fold, while the overall level of HPR1 or THOC5 in the cell remained unchanged, which was evidence for the specificity of ENY2 RNAi (Fig. 4C). However, the association of THOC5 with the hsp 70 gene was significantly weakened and dropped almost to the background level at both the region adjoining the promoter and the downstream region after heat shock (Fig. 4A).

The impairment of THO recruitment may be caused by a general decrease in $h s p 70$ transcription, observed after ENY2 knockdown (MM Kurshakova et al. 2007). Indeed, 
A
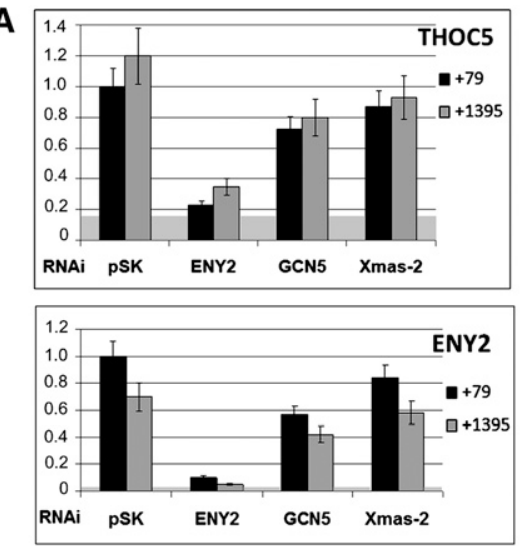

B

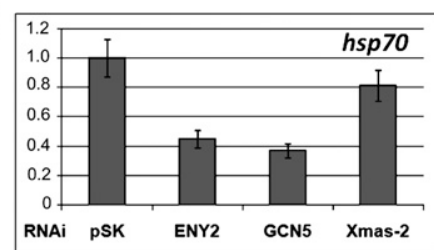

Figure 4. ENY2 knockdown impairs THO recruitment to hsp70. (A) The presence of THOC5 and ENY2 on the hsp70 transcribed region at positions +79 (black bars) and +1395 (gray bars) (see Fig. 3A) following heat shock was evaluated by ChIP after ENY2, GCN5, or Xmas-2 knockdown. In sham experiments, cells were treated with nonspecific dsRNA (pSK). The results of ChIP were normalized to the level of corresponding factor at position +79 . The gray staining shows the baseline level. (B) The level of hsp70 transcript after knockdown of the above factors. $(C)$ Specificity of ENY2, GCN5, and Xmas-2 knockdowns. The presence of the indicated factors was analyzed in cells treated with nonspecific dsRNA (pSK) or corresponding dsRNA. Tubulin was used as a loading control. (D) ENY2 and THO components are not associated with Pol II in cell extract. Immunoprecipitation with antibodies against ENY2, THOC5, HPR1, and Pol II, and with preimmune serum was performed with the extract from S2 cells after heat shock. The precipitate was checked for the presence of total Pol II and Pol II carrying phosphorylated Ser5 in CTD.

the dependence of THO recruitment on the transcription level was described in yeast (Jimeno et al. 2008). To clarify this point, we performed experiments on the depletion of GCN5, which resulted in a general decrease in hsp70 transcription to the level similar to that observed after ENY2 depletion (Fig. 4B). However, the impairment of THO recruitment in this case was moderate, compared with that after ENY2 knockdown. Moreover, THO recruitment remained almost unchanged in the case of Xmas-2 knockdown, which had a slight effect on hsp70 transcription. In general, the effect of GCN5 and Xmas-2 knockdown on THO recruitment is comparable with the influence on hsp70 transcription. Similar data were obtained for the HPR1 subunit of THO (data not shown). We concluded that the significant drop in THO recruitment after ENY2 knockdown is due mainly to the absence of the latter factor rather than to the decrease in hsp70 activity. The behavior of ENY2 after GCN5 and Xmas-2 knockdown was similar to that of THOC5, further corroborating its association with THO in the transcribed region (Fig. 4A). Thus, ENY2 as a component of THO is crucial for its recruitment onto the gene.

To estimate the possible role of Pol II in the recruitment of Drosophila ENY2 and THO to the gene, we performed coimmunoprecipitation (co-IP) experiments with S2 cell nuclear extracts and analyzed whether Pol II was associated with the ENY2-THO complex before or after heat shock. The results are partly shown in Figure 4D. No stable interaction of ENY2, THOC5, or HPR1 with Pol II was revealed in experiments with either antitotal Pol II antibody or antibodies specific to C-terminal domain (CTD) Ser2- or Ser5-phosphorylated Pol II forms. Thus, ENY2 and THO recruitment apparently is not linked directly to the elongating Pol II molecule.

\section{ENY2 and THO are cotranscriptionally loaded onto nascent $m R N A$}

We further tested whether the presence of ENY2 and THO in the transcribed region of the gene was due to their interaction with chromatin or with nascent pre-mRNA. To this end, the assay developed by Abruzzi et al. (2004) was used. In ChIP experiments, chromatin was treated with RNase prior to immunoprecipitation. This treatment eliminated RNA-bound proteins but did not affect proteins directly associated with chromatin.

RNase treatment had only a slight effect on Pol II distribution both on the promoter and in the transcribed region, but strongly reduced the level of HPR1 and THOC5 along the whole gene, with only a minor amount of THO remaining in its $3^{\prime}$ region. This result indicates that the association of THO with the gene is mainly RNA-dependent.

The level of ENY2 on the transcribed region also decreased significantly. However, the peak of ENY2 on the promoter became even higher, suggesting that ENY2 in the promoter region was chromatin-bound. A slight rise of ENY2 and Pol II peaks after RNase treatment may be explained by an increase in the accessibility of these proteins to antibodies after digestion of nascent RNA and associated factors. A similar effect was observed by Abruzzi et al. (2004). The results of experiments with RNase treatment indicate that ENY2 is a component of DNA-bound transcription machinery on the promoter of the gene, while the presence of ENY2 and THO on its transcribed region depends mainly on their binding with nascent mRNA.

To verify ENY2 and THO association with mRNA, we tested whether antibodies against ENY2, THOC5, and HPR1 would coimmunoprecipitate the hsp70 mRNA from the lysate of S2 cells exposed to heat shock (Fig. 5B). These antibodies, as well as those against Xmas-2, specifically coprecipitated the $h s p 70$ mRNA, confirming that ENY2 and Drosophila THO were associated with the hsp70 mRNA particles, as was the case with Xmas-2.

Taken together, our results show that ENY2, THOC5, and HPR 1 are associated with nascent mRNA particles.

We also analyzed the distribution of the THO complex within Drosophila S2 cells. Most of ENY2 and Xmas-2 
A
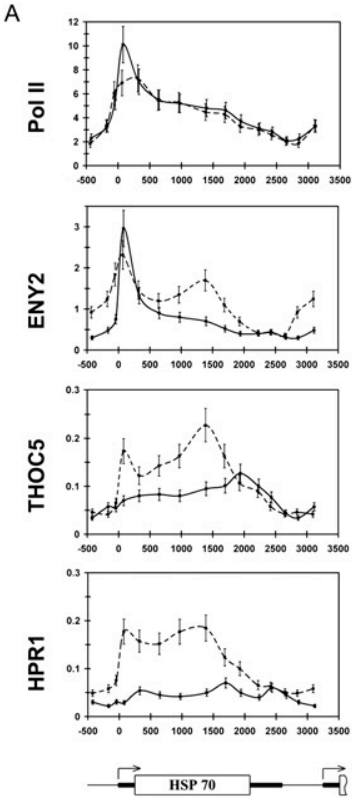

B

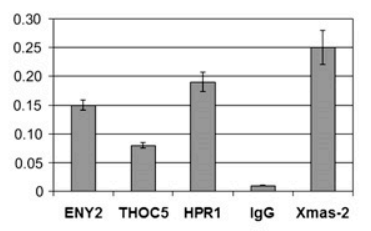

C

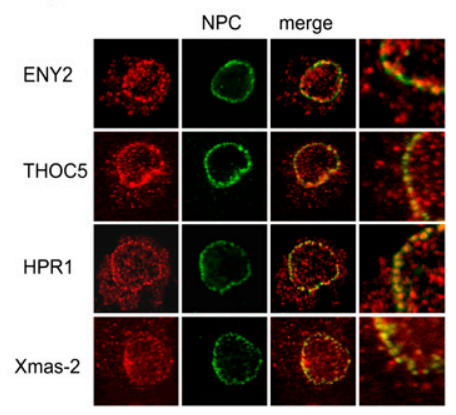

Figure 5. ENY2, THO, and Xmas-2 are associated with mRNA. (A) The effect of RNase treatment on the distribution of Pol II, ENY2, THOC5, and HPR1 on the hsp70 gene after heat shock. The solid line shows the results of experiments in which chromatin was subjected to RNase treatment during the ChIP procedure. The dashed line shows distribution without the treatment (heat shock) (see Fig.3A). The results of ChIP are shown as the percentage of input, with the baseline level varying from $0.02 \%$ to $0.05 \%$ at different points. (B) hsp 70 mRNA was coimmunoprecipitated with ENY2, THO, and Xmas-2. RNA immunoprecipitation was done with indicated antibodies (preimmune IgG was used as a negative control) from the lysate of S2 cells after heat shock. The results are shown as the percentage of input. (C) THO components colocalize with the NPC. Immunostaining of Drosophila S2 cells with antibodies against ENY2, THOC5, HPR1, Xmas-2 (in red), and NPC (in green); merged images; and magnified fragments are shown. Magnification: $1000 \times$; right (merged) images, $3000 \times$.

was visualized as dots at the nuclear periphery and inside the nucleus, as was reported previously (MM Kurshakova et al. 2007). The THO components showed a dotted distribution pattern in the nuclei, similar to that of AMEX subunits (Fig. 5C). Significantly, both THOC5 and HPR1 colocalized with the NPC on the nuclear envelope. It is also noteworthy that weaker signals for all of these proteins were also detected in the cytoplasm of S2 cells.

\section{ENY2 or THO knockdown impairs the 3' processing of hsp70 mRNA}

As shown recently, THO2 or HPR1 mutations in yeast impair mRNA 3'-end processing (Saguez et al. 2008). In this context, it was interesting to study the effect of ENY2 or THO knockdown on the relative level of unprocessed hsp70 mRNA in S2 cells after heat shock (Fig. 6A). The level of unprocessed transcripts was measured by quantitative PCR (qPCR) on a cDNA template synthesized using random primers. For more reliable mea-

surements, we used two different pairs of primers covering the cleavage signal, with the results obtained with either pair being the same (Fig. 6B).

The knockdown of THOC5 and HPR1 resulted in an approximately twofold increase in the level of the uncleaved hsp 70 transcripts; after ENY2 knockdown, this level increased by a factor of $\sim 1.5$ relative to that in the control cells. The knockdown of Xmas-2 or GCN5 had no such effect on 3' processing. Thus, it was ENY2 and THO knockdown that proved to affect $3^{\prime}$ processing of $h s p 70$ transcripts.

\section{Discussion}

Our previous data showed that ENY2 is a chromatinbound protein participating in transcription activation (Georgieva et al. 2001). We demonstrated that ENY2 is a member of both the novel Drosophila mRNA export complex AMEX and the SAGA/TFTC complex. ENY2 concentrates at the nuclear periphery, is associated with the NPC, and has a role in the anchoring of a subset of transcription sites to the NPCs, which provides for efficient transcription and mRNA export (MM Kurshakova et al. 2007).

The data obtained in this study provide evidence that ENY2 is also associated with the THO complex. The results of several independent experiments confirm the significant role of the ENY2-THO interaction in vivo. Firstly, both of these components are distributed along the whole transcribed $h s p 70$ gene, and cotranscriptional recruitment of THO depends on the presence of ENY2.

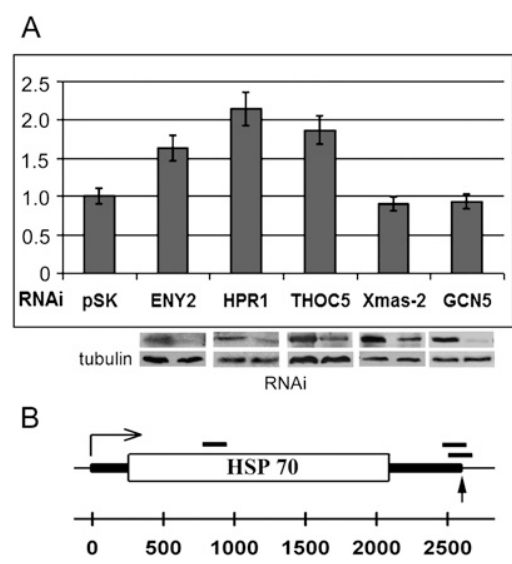

Figure 6. The influence of THO, ENY2, GCN5, and Xmas-2 knockdown on 3 '-end processing of the hsp 70 mRNA. (A) Relative levels of uncleaved hsp70 transcript after knockdown of the above factors. Cells treated with nonspecific dsRNA (pSK) were taken as a control. The levels of corresponding proteins in sham-treated cells (left lanes) or in cells after RNAi treatment (right lanes) are shown below. (B) Scheme of the hsp70 gene, with horizontal bars indicating gene fragments used for estimating the total level of its transcripts and the level of the uncleaved transcript form. Two downstream fragments can be obtained only from an unprocessed transcript, since the cleavage site (arrow at the end of the transcribed region) lies within the fragments. 
Secondly, ENY2 and THO colocalize in many chromosomal sites corresponding to actively transcribed regions. Finally, a strong synergistic effect is observed after combining weak mutations that lead to only a slight decrease in the contents of ENY2 and THO components in flies. A combination of mutations, each causing almost no phenotypic change, results in low viability and strong development retardation.

The above experiments suggest that THO is distributed genome-wide in Drosophila. THO is present in actively transcribed chromatin sites, which is in line with data on yeast and humans (Strasser et al. 2002; Zenklusen et al. 2002; Kim et al. 2004; Masuda et al. 2005). However, THO knockdown affects a limited number of genes in both yeast and Drosophila cell cultures (Rehwinkel et al. 2004; Rougemaille et al. 2008), which may be explained by the existence of a redundant system masking THO depletion.

In the inactive hsp70 gene, ENY2 is present on the promoter, similarly to other SAGA components (Lebedeva et al. 2005; MM Kurshakova et al. 2007). Following transcription activation, ENY2 is recruited onto the whole transcribed region, as is the THO complex, with the distribution profiles of THO and ENY2 being almost identical. In line with the result obtained in yeast (Kim et al. 2004), we did not detect THO or ENY2 in the region downstream from the poly(A) site. The results of ChIP with RNase treatment and RNA immunoprecipitation experiments show that Drosophila ENY2 and THO bind mainly to nascent mRNA during transcription. An exception is the peak of ENY2 at the hsp70 promoter, where ENY2 is chromatin-bound. The observed association of THO with mRNA rather than with chromatin, as is the case with yeasts (Huertas and Aguilera 2003; Abruzzi et al. 2004), further confirms this metazoan-specific feature of THO (Masuda et al. 2005). Our results also confirm that ENY2 is important for proper association of THO with nascent mRNA.

THO components show two peaks in the transcribed region. The peak located immediately downstream from the promoter appears to reflect the interaction of THO with the cap, which was demonstrated for the human TREX complex (Cheng et al. 2006). The second peak of THO is located within the transcribed region of the gene and may be accounted for by the interaction of THO with other mRNA-associated factors or mRNA itself (Masuda et al. 2005; Katahira et al. 2009). Recruitment of yeast THO onto the transcribed region was shown to be Pol IIdependent (Abruzzi et al. 2004). Moreover, Sus1, the yeast homolog of ENY2, is associated with the elongating form of Pol II (Pascual-Garcia et al. 2008). However, we detected no stable association of either ENY2 or THO with Pol II, which indicates that the recruitment of THO to nascent transcripts in Drosophila is not linked directly to elongating Pol II. This is one more specific feature of the metazoan THO complex (Masuda et al. 2005) corroborated in our study.

The depletion of THO subunits or ENY2 leads to an increase in the proportion of transcripts with unprocessed $3^{\prime}$ ends. The same phenotype was described for THO mutants in yeast (Rougemaille et al. 2008; Saguez et al.
2008). It was suggested that the lack of functional THO leads to improper mRNP packaging, with consequent partial destruction of the $3^{\prime}$-end processing machinery (Saguez et al. 2008). Our data indicate that this phenotype of THO knockdown may be common to yeasts and metazoans.

We also compared the involvement of the Xmas-2 subunit of AMEX and THO in mRNP formation. In our previous study (MM Kurshakova et al. 2007), Xmas-2 was found to be recruited to the loci of active transcription. Here, we showed that Xmas-2 is associated with mRNA. However, conventional ChIP fails to reveal Xmas-2 association with chromatin of an active gene, in contrast to the situation with THO. Therefore, it appears that Xmas-2 is loaded onto mRNA regions located fairly distantly from the chromatin template. In contrast, the ENY2-THO complex appears to operate in close proximity to chromatin, which may account for its important role in preventing RNA-DNA hybrid formation in yeast (Huertas and Aguilera 2003). We also revealed no influence of Xmas-2 knockdown on $3^{\prime}$-end processing. Functions of Xmas-2 apparently pertain to later stages of formation and export of mRNPs (MM Kurshakova et al. 2007). Studies on yeasts also suggest a role for the Xmas-2 homolog Sac3 in the post-transcriptional maturation of nuclear mRNA, in addition to its role in mRNA export (Chekanova et al. 2008; Gonzalez-Aguilera et al. 2008).

We obtained several lines of evidence that the ENY2THO complex exists and functions, mainly independently, on other ENY2-containing complexes; namely, SAGA and AMEX. The THO complex is not associated with SAGA or AMEX subunits in the nuclear extract. Genetically, SAGA and THO mutations have different effects on phenotypic manifestations of the ENY2 mutation, suggesting that the spectra of genes under the control of ENY2-THO and ENY2-SAGA do not completely coincide. At the molecular level, we detected no direct association of THO with elongating Pol II. This fact is in favor of the model that ENY2-THO recruitment to the transcribed region is not connected with the promoter and promoter-associated SAGA, but rather occurs directly on nascent mRNA.

The functioning of ENY2, as well as of THO and Xmas-2, is apparently not confined to the nucleus, since we also detected a significant amount of these factors in the cytoplasm of S2 cells. THO shuttling between the nucleus and cytoplasm was shown for humans (Katahira et al. 2009). The Thp1-Sac3 complex was found at the cytoplasmic face of the NPC (Fischer et al. 2002). These observations indicate that ENY2 and other factors studied may also be of significance for the fate of mRNPs and their functioning in the cytoplasm.

The data available today confirm that ENY2, being a component of different protein complexes, is involved in several sequential steps of the gene expression process in the nucleus (Fig. 7). We suggest that the role of ENY2 may be related to proper spatial coordination of the recruitment of these complexes onto DNA or RNA, as well as to gene positioning in the nucleus. A similar function was proposed for the Yral protein, which has 


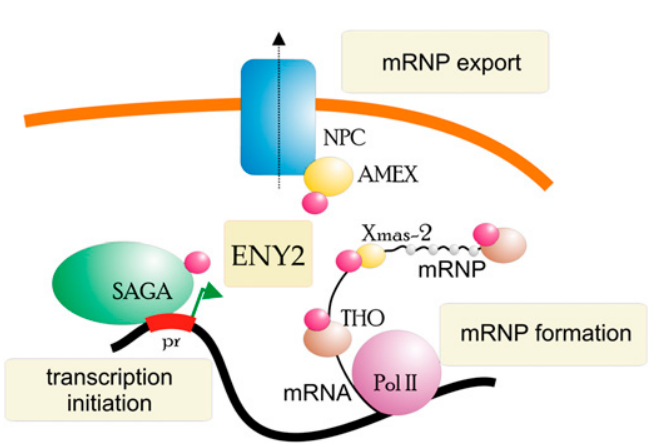

Figure 7. ENY2 participates in consecutive steps of gene expression in the nucleus. ENY2 as a component of SAGA is localized on the promoter of the gene, also participating in proper gene positioning in the nucleus. During transcription, ENY2 in a complex with THO is loaded onto the growing transcript in proximity to chromatin, with this process apparently occurring without direct participation of Pol II. THO and ENY2 are distributed along the whole transcript, starting from its $5^{\prime}$ end. The Xmas-2 factor is also loaded onto mRNA at a later stage, distantly form chromatin. The presence of ENY2 and THO is necessary for proper 3 '-end processing and export of mRNA. ENY2 and Xmas-2 are also components of the AMEX mRNA export complex, which is associated with the NPC.

a number of protein partners (Bruhn et al. 1997; Iglesias and Stutz 2008). Thus, there may well be a class of factors that serve to integrate the complex process of gene expression.

In summary, our findings further clarify the complicated picture of mRNP formation and export in metazoans. We analyzed specific features of the metazoan THO complex in more detail and showed that the ENY2 transcription factor orchestrates consecutive steps of gene expression in the nucleus.

\section{Materials and methods}

\section{Antibodies}

We used antibodies against ENY2 (Georgieva et al. 2001), Xmas-2 (MM Kurshakova et al. 2007), Pol II (Georgieva et al. 2000), Pol IIS5P and Pol II-S2P (Abcam), GCN5 (Lebedeva et al. 2005), and TBP (Vorobyeva et al. 2009). Antibodies against HPR1 (fragment, 60-223 amino acids) and THOC5 (fragment, 1-219 amino acids) were raised in our laboratory by immunizing rabbits with the corresponding His $_{6}$-tagged protein fragments. All rabbit antibodies were affinity-purified. An antibody against $\beta$-tubulin, obtained by M. Klymkowsky, was from the Developmental Studies Hybridoma Bank, developed under the auspices of the NICHD, and maintained at the Department of Biological Sciences, University of Iowa.

\section{Drosophila genetic crosses}

Tho2 (11106) and Thoc6 (17839) mutant fly strains were obtained from the Bloomington Stock Center. The GCN5 $5^{\text {C137T }}$ (hypomorphic) and GCN5 ${ }^{\text {E33sst }}$ (null) GCN5 mutations (Carre et al. 2005) and the $e(y) 2^{u 1}$ mutation (Georgieva et al. 2001) were described previously. $e(y) 2^{u 1} / F M 4$ females were crossed with males carrying autosomal mutations Tho2 or Thoc6, or with GCN5 ${ }^{C 137 T} / T M$ or GCN5 $5^{E 333 s t} / T M 33$ males. In the F1 progeny, males hemizygous for $e(y) 2^{u 1}$ were examined for morphological defects. The survival rate was calculated as the percent ratio of males with a given genotype to males carrying the corresponding balancer chromosome. All genetic crosses were performed at $25^{\circ} \mathrm{C}$ in at least three replications. No fewer than 40 flies of each viable genotype were screened.

\section{Purification of ENY2-containing complexes and immunoprecipitation}

ENY2-containing complexes were purified from nuclear extracts of 0- to 12-h Drosophila embryos. Details of the purification procedure are shown in Figure 1B. The columns were equilibrated with the HEMG buffer (25 mM HEPES-KOH at $\mathrm{pH} 7.6$, $12.5 \mathrm{mM} \mathrm{MgCl} 2,0.1 \mathrm{mM}$ EDTA, 10\% glycerol, $1 \mathrm{mM} \mathrm{DTT)}$ containing $150 \mathrm{mM} \mathrm{NaCl}$ (HEMG-150). Immunoaffinity purification was performed on a column with immobilized anti-ENY2 antibodies, with unbound protein being washed out with HEMG-1000 buffer containing $0.1 \%$ NP-40. For other details of the purification procedure and MALDI-TOF MS experiments, see Vorobyeva et al. (2009). The Superose 6 column was calibrated with an HMW Calibration Kit (GE Healthcare). The void volume of the column was $7.0 \mathrm{~mL}$, and the volume of each fraction was $0.5 \mathrm{~mL}$.

Preparation of embryonic nuclear extracts and immunoprecipitation were performed as described (Georgieva et al. 2000). Proteins from S2 cells were extracted in a lysis buffer $10 \mathrm{mM}$ HEPES at $\mathrm{pH} 7.9,5 \mathrm{mM} \mathrm{MgCl}_{2}, 0.5 \% \mathrm{NP}-40,0.45 \mathrm{M} \mathrm{NaCl}, 1 \mathrm{mM}$ DTT) containing the complete protease inhibitor cocktail (Roche) and phosphatase inhibitors cocktails 1 and 2 (Sigma). Prior to immunoprecipitation, the extract was treated with DNase I (USB, $0.6 \mathrm{U} / \mathrm{mL}$ ) and RNase (Stratagene, $10 \mathrm{U} / \mathrm{mL}$ ).

\section{Drosophila cell culture and RNAi knockdown experiments}

Drosophila S2 cells were cultured at $25^{\circ} \mathrm{C}$ in Schneider's insect medium (Sigma) containing 10\% fetal bovine serum (HyClone).

RNAi experiments followed the published protocol (Clemens et al. 2000). We used 15-20 $\mu$ g of dsRNA per $1 \times 10^{6}$ cells; dsRNA was synthesized with an Ambion MEGA Script T7 kit. The expression of the target genes was measured by qPCR and Western blot analysis. dsRNA corresponding to a fragment of pBluescript II (pSK) vector was used as a negative control. The following primers were used for the synthesis: THOC5, 5'-CGA CTCACTATAGGGAGACGCCAACCGCGAGGTGAAGC-3' and $5^{\prime}$-CGACTCACTATAGGGAGAGGAGCGAGTGAGTCG TCCAAGTTG-3'; HPR1，5'-CGACTCACTATAGGGAGAGA CGGCAAAGTGGAGCTGTT-3' and 5'-CGACTCACTATAGG GAGATCCACTGCGGCTTATTGTAACA-3'. Primers for RNAi of ENY2 and Xmas-2 were as described (MM Kurshakova et al. 2007).

\section{ChIP and $q P C R$ analysis}

ChIP was performed according to the published procedure (Boehm et al. 2003). DNA was cross-linked (1\% FA, $15 \mathrm{~min}$ ) and sheared to a size of $\sim 300$ base pairs (bp). Approximately $3 \times$ $10^{6}$ cells and $10 \mu \mathrm{g}$ of an antibody were taken for one experiment. After ChIP, the recovered DNA was analyzed by qPCR with MiniOpticon (Bio-Rad). Measurements were made at 13 points within the $h s p 70$ gene; namely, at positions $-422,-168,-43,79$, $188,329,648,974,1395,1698,1940,2434$, and $2664 \mathrm{bp}(+1$ is the transcription start point). Sequences of the primers used are available on request. Each point was measured in at least five experiments, and the mean value was calculated. For baseline (control) measurements, ChIP with preimmune IgG was used. In experiments with RNase treatment, RNase A (Fermentas, $30 \mu \mathrm{g}$ 
per experiment) was added $30 \mathrm{~min}$ prior to incubation with antibodies.

\section{RNA immunoprecipitation}

Co-IP of $h s p 70$ mRNA with antibodies was performed as described (Tenenbaum et al. 2000; Baroni et al. 2008) from the lysate of S2 cells exposed to heat shock for $20 \mathrm{~min}$ at $37^{\circ} \mathrm{C}$. No cross-linking reagent was used. For immunoprecipitation, the antibody-protein A Sepharose beads were incubated with the lysate for $1 \mathrm{~h}$ at $4^{\circ} \mathrm{C}$ in NT2 buffer $(50 \mathrm{mM}$ Tris- $\mathrm{HCl}$ at $\mathrm{pH} 7.4$, $150 \mathrm{mM} \mathrm{NaCl}, 1 \mathrm{mM} \mathrm{MgCl} 2,0.05 \% \mathrm{NP}-40$ ) containing $4 \mathrm{U} / \mu \mathrm{L}$ RiboLock (Fermentas), 1\% BSA, and $1 \mathrm{mg} / \mathrm{mL}$ ssDNA. After washing, the TRI Reagent (Ambion) was added to extract RNA. For reverse transcription, oligo-dT primer was used. The level of hsp70 mRNA was measured by qPCR using primers $5^{\prime}$-TTGG GCGGCGAGGACTTTG-3' and 5'-GCTGTTCTGAGGCGTC GTAGG-3'. Each experiment was performed in at least three replications, calculating the mean value.

\section{Measuring the level of hsp70 transcript with unprocessed $3^{\prime}$ ends}

After heat shock $\left(20 \mathrm{~min}, 37^{\circ} \mathrm{C}\right)$, S2 cell nuclei were isolated using LB buffer $(10 \mathrm{mM}$ Tris- $\mathrm{HCl}$ at $\mathrm{pH} 7.8,10 \mathrm{mM} \mathrm{NaCl}$, $1.5 \mathrm{mM} \mathrm{MgCl}_{2}, 1 \mathrm{mM} \mathrm{DTT}$ ). RNA from the nuclei was extracted with an RNeasy Mini Plus Kit (Qiagen). To avoid contamination with genomic DNA, the preparation was treated with DNase I. The amount of RNA was measured with a Qubit fluorometer (Invitrogen). Equal amounts of RNA and random primers were used to produce cDNA probes, with their contents being measured by qPCR. Two pairs of primers to the nonprocessed transcript of the $h s p 70$ gene were used: $5^{\prime}$-GTTGGCATCCCT ATTAAACAGC-3' and 5'-CAGGACTCACTTAGCGGGG-3'; $5^{\prime}$-ATTACGGATTACAAAATGGAACC-3' and 5' ${ }^{\prime}$-AATTGGG ATGACGGATCTC-3'. The total hsp70 transcript level was measured with primers indicated in the "RNA Immunoprecipitation" section above. The ratio of unprocessed-to-total transcript level was calculated.

\section{Immunostaining}

Immunostaining of S2 cells and polytene chromosomes was performed as described (Soldatov et al. 1999) using mouse antiENY2, rabbit anti-THOC5, and corresponding secondary antibodies (Molecular Probes).

\section{Acknowledgments}

We are grateful to A. Ivliev for his assistance in raising antibodies, I. Toropygin for his assistance with MALDI experiments, and N.A. Gorgolyuk for his help in preparing the manuscript. GCN5 mutations were a kind gift from Christophe Antoniewski. D.K. was supported by the Young Scientists Support Program of the President of the Russian Federation (project no. MK-3564.2009.4). Y.S. and A.K. acknowledge a fellowship from University of Oslo, Centre for Medical Studies, in Russia. This study was supported by the program "Molecular and Cell Biology" of the Russian Academy of Sciences, RFBR grant number 07-04-01076, and Scientific School Support grant number 2994.2008.4.

\section{References}

Abruzzi KC, Lacadie S, Rosbash M. 2004. Biochemical analysis of TREX complex recruitment to intronless and introncontaining yeast genes. EMBO J 23: 2620-2631.
Baroni TE, Chittur SV, George AD, Tenenbaum SA. 2008. Advances in RIP-chip analysis: RNA-binding protein immunoprecipitation-microarray profiling. Methods Mol Biol 419: 93-108.

Boehm AK, Saunders A, Werner J, Lis JT. 2003. Transcription factor and polymerase recruitment, modification, and movement on dhsp70 in vivo in the minutes following heat shock. Mol Cell Biol 23: 7628-7637.

Bruhn L, Munnerlyn A, Grosschedl R. 1997. ALY, a contextdependent coactivator of LEF-1 and AML-1, is required for TCR $\alpha$ enhancer function. Genes \& Dev 11: 640-653.

Cabal GG, Genovesio A, Rodriguez-Navarro S, Zimmer C, Gadal O, Lesne A, Buc H, Feuerbach-Fournier F, Olivo-Marin JC, Hurt EC, et al. 2006. SAGA interacting factors confine sub-diffusion of transcribed genes to the nuclear envelope. Nature 441: 770-773.

Carre C, Szymczak D, Pidoux J, Antoniewski C. 2005. The histone $\mathrm{H} 3$ acetylase dGCN5 is a key player in Drosophila melanogaster metamorphosis. Mol Cell Biol 25: 8228-8238.

Chavez S, Beilharz T, Rondon AG, Erdjument-Bromage $\mathrm{H}$, Tempst P, Svejstrup JQ, Lithgow T, Aguilera A. 2000. A protein complex containing Tho2, Hpr1, Mft1 and a novel protein, Thp2, connects transcription elongation with mitotic recombination in Saccharomyces cerevisiae. EMBO J 19: 5824-5834.

Chekanova JA, Abruzzi KC, Rosbash M, Belostotsky DA. 2008. Sus1, Sac3, and Thp1 mediate post-transcriptional tethering of active genes to the nuclear rim as well as to non-nascent mRNP. RNA 14: 66-77.

Cheng H, Dufu K, Lee CS, Hsu JL, Dias A, Reed R. 2006. Human mRNA export machinery recruited to the $5^{\prime}$ end of mRNA. Cell 127: 1389-1400.

Clemens JC, Worby CA, Simonson-Leff N, Muda M, Maehama T, Hemmings BA, Dixon JE. 2000. Use of double-stranded RNA interference in Drosophila cell lines to dissect signal transduction pathways. Proc Natl Acad Sci 97: 6499-6503.

Demeny MA, Soutoglou E, Nagy Z, Scheer E, Janoshazi A, Richardot M, Argentini M, Kessler P, Tora L. 2007. Identification of a small TAF complex and its role in the assembly of TAF-containing complexes. PLoS One 2: e316. doi: 10.1371/ journal.pone.0000316.

Fischer T, Strasser K, Racz A, Rodriguez-Navarro S, Oppizzi M, Ihrig P, Lechner J, Hurt E. 2002. The mRNA export machinery requires the novel Sac3p-Thp1p complex to dock at the nucleoplasmic entrance of the nuclear pores. $E M B O ~ I ~ 21:$ 5843-5852.

Georgiev PG, Gerasimova TI. 1989. Novel genes influencing the expression of the yellow locus and mdg4 (gypsy) in Drosophila melanogaster. Mol Gen Genet 220: 121-126.

Georgieva S, Kirschner DB, Jagla T, Nabirochkina E, Hanke S, Schenkel H, de Lorenzo C, Sinha P, Jagla K, Mechler B, et al. 2000. Two novel Drosophila TAF(II)s have homology with human TAF(II)30 and are differentially regulated during development. Mol Cell Biol 20: 1639-1648.

Georgieva S, Nabirochkina E, Dilworth FJ, Eickhoff H, Becker P, Tora L, Georgiev P, Soldatov A. 2001. The novel transcription factor e/y)2 interacts with $\mathrm{TAF}(\mathrm{II}) 40$ and potentiates transcription activation on chromatin templates. Mol Cell Biol 21: 5223-5231.

Gonzalez-Aguilera C, Tous C, Gomez-Gonzalez B, Huertas P, Luna R, Aguilera A. 2008. The THP1-SAC3-SUS1-CDC31 complex works in transcription elongation-mRNA export preventing RNA-mediated genome instability. Mol Biol Cell 19: 4310-4318.

Huertas P, Aguilera A. 2003. Cotranscriptionally formed DNA:RNA hybrids mediate transcription elongation 
impairment and transcription-associated recombination. Mol Cell 12: 711-721.

Iglesias N, Stutz F. 2008. Regulation of mRNP dynamics along the export pathway. FEBS Lett 582: 1987-1996.

Jani D, Lutz S, Marshall NJ, Fischer T, Kohler A, Ellisdon AM, Hurt E, Stewart M. 2009. Sus1, Cdc31, and the Sac3 CID region form a conserved interaction platform that promotes nuclear pore association and mRNA export. Mol Cell 33: 727-737.

Jimeno S, Rondon AG, Luna R, Aguilera A. 2002. The yeast THO complex and mRNA export factors link RNA metabolism with transcription and genome instability. EMBO J 21: 3526-3535.

Jimeno S, Luna R, Garcia-Rubio M, Aguilera A. 2006. Tho1, a novel hnRNP, and Sub2 provide alternative pathways for mRNP biogenesis in yeast THO mutants. Mol Cell Biol 26: 4387-4398.

Jimeno S, Garcia-Rubio M, Luna R, Aguilera A. 2008. A reduction in RNA polymerase II initiation rate suppresses hyper-recombination and transcription-elongation impairment of THO mutants. Mol Genet Genomics 280: 327-336.

Katahira J, Inoue H, Hurt E, Yoneda Y. 2009. Adaptor Aly and coadaptor Thoc5 function in the Tap-p15-mediated nuclear export of HSP70 mRNA. EMBO I 28: 556-567.

Kim M, Ahn SH, Krogan NJ, Greenblatt JF, Buratowski S. 2004. Transitions in RNA polymerase II elongation complexes at the 3' ends of genes. EMBO I 23: 354-364.

Kohler A, Pascual-Garcia P, Llopis A, Zapater M, Posas F, Hurt E, Rodriguez-Navarro S. 2006. The mRNA export factor Sus1 is involved in Spt/Ada/GCN5 acetyltransferase-mediated H2B deubiquitinylation through its interaction with Ubp8 and Sgf11. Mol Biol Cell 17: 4228-4236.

Krasnov AN, Kurshakova MM, Ramensky VE, Mardanov PV, Nabirochkina EN, Georgieva SG. 2005. A retrocopy of a gene can functionally displace the source gene in evolution. Nucleic Acids Res 33: 6654-6661.

Kurshakova M, Maksimenko O, Golovnin A, Pulina M, Georgieva S, Georgiev P, Krasnov A. 2007. Evolutionarily conserved E/y)2/ Sus1 protein is essential for the barrier activity of $\mathrm{Su}(\mathrm{Hw})$ dependent insulators in Drosophila. Mol Cell 27: 332-338.

Kurshakova MM, Krasnov AN, Kopytova DV, Shidlovskii YV, Nikolenko JV, Nabirochkina EN, Spehner D, Schultz P, Tora L, Georgieva SG. 2007. SAGA and a novel Drosophila export complex anchor efficient transcription and mRNA export to NPC. EMBO J 26: 4956-4965.

Kusch T, Guelman S, Abmayr SM, Workman JL. 2003. Two Drosophila Ada2 homologues function in different multiprotein complexes. Mol Cell Biol 23: 3305-3319.

Lebedeva LA, Nabirochkina EN, Kurshakova MM, Robert F, Krasnov AN, Evgen'ev MB, Kadonaga JT, Georgieva SG, Tora L. 2005. Occupancy of the Drosophila hsp70 promoter by a subset of basal transcription factors diminishes upon transcriptional activation. Proc Natl Acad Sci 102: 1808718092.

Masuda S, Das R, Cheng H, Hurt E, Dorman N, Reed R. 2005. Recruitment of the human TREX complex to mRNA during splicing. Genes \& Dev 19: 1512-1517.

Mohrmann L, Verrijzer CP. 2005. Composition and functional specificity of SWI2/SNF2 class chromatin remodeling complexes. Biochim. Biophys. Acta-Gene Struct. Exp. 1681: 5973.

Pascual-Garcia P, Govind CK, Queralt E, Cuenca-Bono B, Llopis A, Chavez S, Hinnebusch AG, Rodriguez-Navarro S. 2008. Sus1 is recruited to coding regions and functions during transcription elongation in association with SAGA and TREX2. Genes \& Dev 22: 2811-2822.
Rasmussen EB, Lis JT. 1993. In vivo transcriptional pausing and cap formation on three Drosophila heat shock genes. Proc Natl Acad Sci 90: 7923-7927.

Rehwinkel J, Herold A, Gari K, Kocher T, Rode M, Ciccarelli FL, Wilm M, Izaurralde E. 2004. Genome-wide analysis of mRNAs regulated by the THO complex in Drosophila melanogaster. Nat Struct Mol Biol 11: 558-566.

Rodriguez-Navarro S, Fischer T, Luo MJ, Antunez O, Brettschneider S, Lechner J, Perez-Ortin JE, Reed R, Hurt E. 2004. Sus1, a functional component of the SAGA histone acetylase complex and the nuclear pore-associated mRNA export machinery. Cell 116: 75-86.

Rougemaille M, Dieppois G, Kisseleva-Romanova E, Gudipati RK, Lemoine S, Blugeon C, Boulay J, Jensen TH, Stutz F, Devaux F, et al. 2008. THO/Sub2p functions to coordinate 3 '-end processing with gene-nuclear pore association. Cell 135: $308-321$.

Saguez C, Schmid M, Olesen JR, Ghazy MA, Qu X, Poulsen MB, Nasser T, Moore C, Jensen TH. 2008. Nuclear mRNA surveillance in $\mathrm{THO} /$ sub2 mutants is triggered by inefficient polyadenylation. Mol Cell 31: 91-103.

Soldatov A, Nabirochkina E, Georgieva S, Belenkaja T, Georgiev P. 1999. TAFII40 protein is encoded by the e(y)1 gene: Biological consequences of mutations. Mol Cell Biol 19: 3769-3778.

Strasser K, Masuda S, Mason P, Pfannstiel J, Oppizzi M, Rodriguez-Navarro S, Rondon AG, Aguilera A, Struhl K, Reed R, et al. 2002. TREX is a conserved complex coupling transcription with messenger RNA export. Nature 417: 304308.

Tenenbaum SA, Carson CC, Lager PJ, Keene JD. 2000. Identifying mRNA subsets in messenger ribonucleoprotein complexes by using cDNA arrays. Proc Natl Acad Sci 97: 1408514090.

Vorobyeva NE, Soshnikova NV, Nikolenko JV, Kuzmina JL, Nabirochkina EN, Georgieva SG, Shidlovskii YV. 2009. Transcription coactivator SAYP combines chromatin remodeler Brahma and transcription initiation factor TFIID into a single supercomplex. Proc Natl Acad Sci 106: 1104911054.

Zenklusen D, Vinciguerra P, Wyss JC, Stutz F. 2002. Stable mRNP formation and export require cotranscriptional recruitment of the mRNA export factors Yralp and Sub2p by Hprlp. Mol Cell Biol 22: 8241-8253.

Zhao Y, Lang G, Ito S, Bonnet J, Metzger E, Sawatsubashi S, Suzuki E, Le Guezennec X, Stunnenberg HG, Krasnov A, et al. 2008. A TFTC/STAGA module mediates histone H2A and $\mathrm{H} 2 \mathrm{~B}$ deubiquitination, coactivates nuclear receptors, and counteracts heterochromatin silencing. Mol Cell 29: 92-101. 


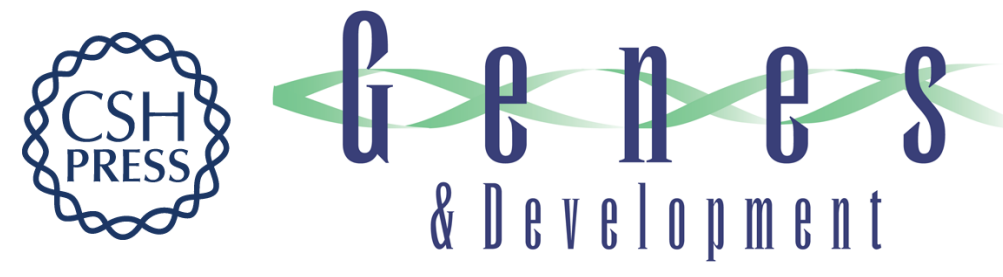

\section{Multifunctional factor ENY2 is associated with the THO complex and promotes its recruitment onto nascent mRNA}

Daria V. Kopytova, Anastasija V. Orlova, Alexey N. Krasnov, et al.

Genes Dev. 2010, 24:

Access the most recent version at doi:10.1101/gad.550010

Supplemental http://genesdev.cshlp.org/content/suppl/2009/12/14/24.1.86.DC1
Material

References This article cites 44 articles, 24 of which can be accessed free at: http://genesdev.cshlp.org/content/24/1/86.full.html\#ref-list-1

License

Email Alerting

Service

Receive free email alerts when new articles cite this article - sign up in the box at the top right corner of the article or click here.

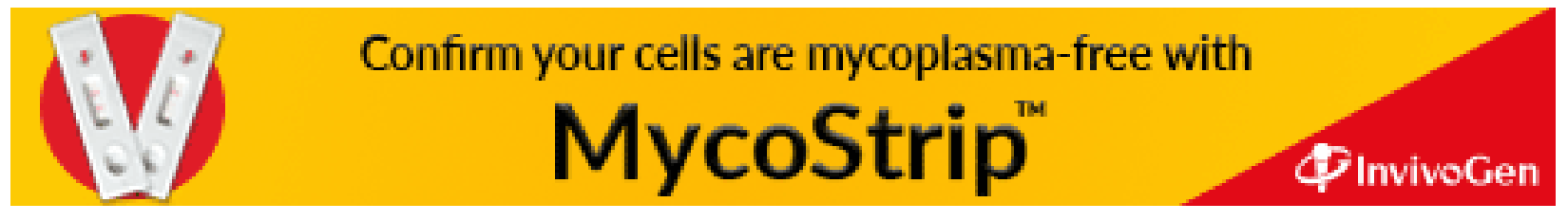

